\title{
The Experimental Study on Thermal Stability of Aqueous Surfactant Solution
}

\author{
Xinnan Song \\ School of Energy and Power Engineering \\ Jiangsu University \\ Zhenjiang, China \\ ekse@,ujs.edu.cn
}

\author{
Yu Wang \\ School of Energy and Power Engineering \\ Jiangsu University \\ Zhenjiang, China \\ 947103749@qq.com
}

\begin{abstract}
Based on the determination of the equilibrium surface tension for anionic surfactant SDS and non-ionic surfactant TrionX-100 and TrionX-114 under different concentrations, the two surfactant continuous boiling test and the SDS for the hydrolysis tests were carried out. Objective is to analyse the variation process for active ingredient of the surfactants. Results show that the liquid with surfactant is capable of reducing the surface tension. With the increment of surfactant concentration, the surface tension decreases gradually, and the surface tension is minimized under the critical micelle concentration(CMC); When the concentration lower than CMC, the surface-active of Triton $\mathrm{X}-100$ presented is stronger than the SDS. At continuous boiling experiment, the thermal stability of Triton $\mathrm{X}-100$ solution is better than the SDS. When the SDS aqueous solution is in the sealed environment with set temperature and the continuous boiling environment under experiment situation, the active ingredients of SDS solution are change and the equilibrium surface tension is undermined. Owing to the chemical losses, the inherent characteristics of solution perform dramatically different from the original one during boiling experiment, which includes the hydrolytic destruction and micro-layer pyrolysis chemical effects.
\end{abstract}

Keywords-surfactants; boiling heat transfer; the equilibrium surface tension; chemical losses; heat flux

\section{INTRODUCTION}

With the energy consumption, people are increasingly aware of the importance of energy conservation. Boiling heat transfer enhancement in the industrial sector is widely used[1-3], the surfactant additive enhancement of boiling heat transfer has been paid the increasing attention due to his less adding, simple operation and strengthen good effect[4-7]. With water steam generated when water boils, and adding a surfactant in water, the surfactant is dissolved in water, an aqueous solution which will inevitably lead to vapor generated during boiling away part of the surface active agents[8-9]. In this paper, different concentrations of anionic surfactant SDS, the non-ionic surfactant Triton X-100 and Triton X-114 solution at different boiling condensation heat flux experiment while collecting the corresponding condensate, and then experimentally measured and calculated corresponding to the concentration of the condensate surfactant.

\section{EQUIPMENT AND METHODS}

Evaporation condensation experimental apparatus shown in Fig. 1 comprises a heating section, boiling section and condenser section. Heating segment mainly composed of the regulator and the heat conducting member. Conditions of this experiment was different heat flux, the heating voltage can be adjusted to change the heat flux boiling pool boiling section made of plexiglass. The condensing section with a snake-type condenser, condenser horizontal tilt $20^{\circ}$ with iron sets fixed. The steam generated by boiling a catheter introduced into the condenser through the glass, by the cooling water through condensation up device, within the steam condenser cooled to liquid, and then the flask was collected condensate flowing down the condense.

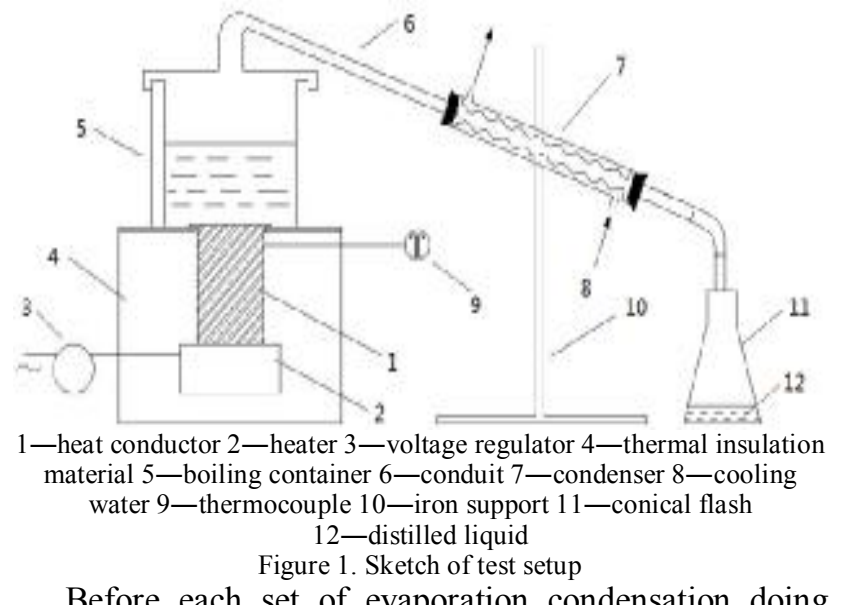
experiments with pure water for the condenser must be cleaned and dried and the conduit in an oven, which is mainly to prevent the accuracy of the influence of impurities and water into the experimental data. Specific experimental procedure is as follows:

1. First previously prepared surfactant solution for cleaning and wetting at the boiling pool, a certain amount of the solution was then poured into boiling pool, and then the heating voltage to the set value to the solution was heated.

2. Observing the boiling phenomena of solution and measuring the temperature of heating wall, when the value reaches a relatively stable, and then evaporation through conduit access vapor condenser, 
the condenser through the cooling water, the flask condensate collected.

3. Sealing the condensate and keeping. When such liquid temperature is down to room temperature $\left(25^{\circ} \mathrm{C}\right)$, measuring the tension by the micro-controlled automatic interfacial tension meter.

\section{EXPERIMENTAL RESULTS AND ANALYSIS}

\section{A. Experimental Data Processing}

The surface tension experimental data of different concentrations solution of SDS, Triton X-100 and Triton X-114 previously measured are used to graph the map which the tension of the vertical axis to graph the concentration of abscissa, and fitting a trend line on the map and correlations. Concentration of the condensate according to anti Deriving its measured surface tension generation fitting correlation. Concentration then calculated based on the condensate and make boiling solution concentration curve.

The Fig. 2 shows the SDS, Triton X-100 and Triton X114 concentration and the surface tension of the aqueous solution of the fitted curve and the correlation. As can be seen from the three figures, these three surfactant concentration and the surface tension of both X-100 exponential relationship, the coefficient in front of the variable $\mathrm{x}$ : Triton $\mathrm{X}-114>$ Triton $\mathrm{X}-100>$ SDS, namely the more surfactants reduce surface tension, the coefficient in front of the larger variable $\mathrm{x}$.

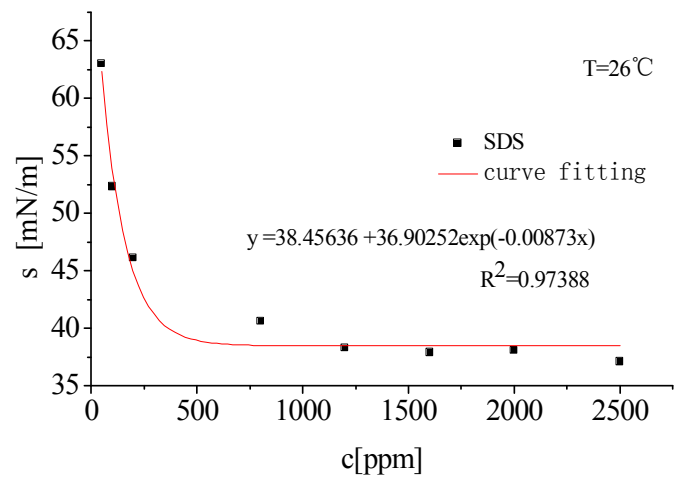

(a)SDS

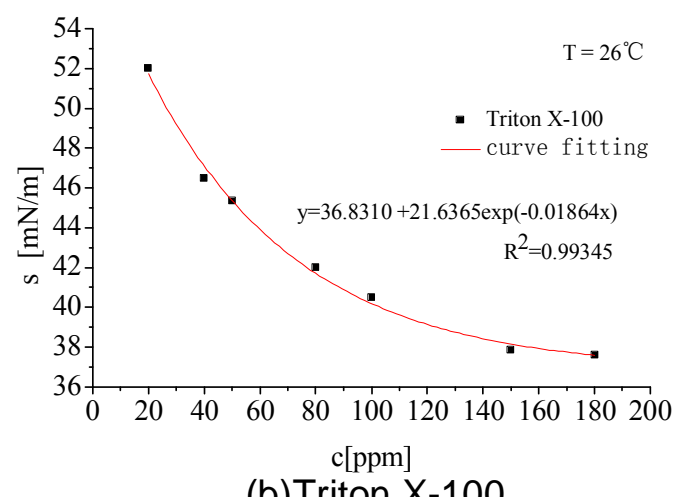

(b) Triton X-100

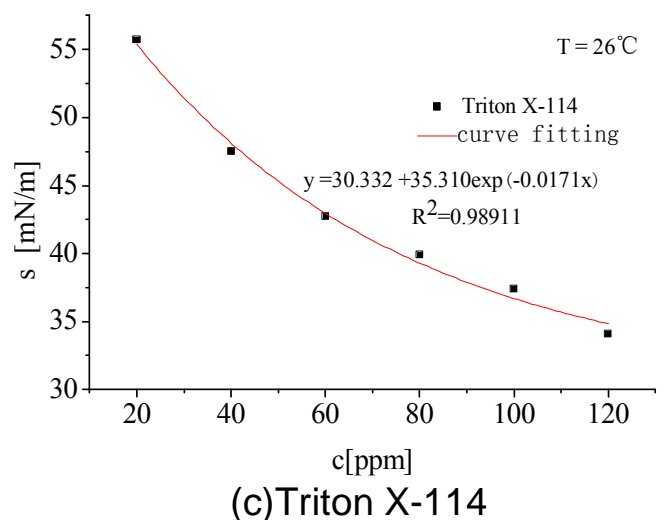

Figure 2. The fitting curve of SDS(a), TritonX-100(b), TritonX$114(\mathrm{c})$ concentration and the surface tension

\section{B. Experimental Results}

The relationship between SDS boiling solution and condensate is shown in Fig.2(a). As can be seen from the figure, the same concentration of the solution under boiling conditions, SDS condensate heat flux increases as the concentration is raised, and a high heat flux increasing trend is particularly significant. When the heat flux density is the same, SDS concentration of the condensate with the overall trend in boiling solution concentration increases, but the concentration anomalies appear in the $\mathrm{CMC}[10]$ ( critical micelle concentration) : condensate presents the maximum concentration.

The relationship between Triton X-100 boiling solution and condensate is shown in Fig.2(b). As can be seen from the figure, under the same concentration of the boiling solution, the concentration of Triton X-100 condensate rises with the increasing heat density, and a high heat flux increasing trend is particularly significant, like the SDS. In the same heat flux density, Triton X-100 concentration condensate boiling solution with increasing concentration large, but also when the concentration CMC performance anomalies: contrast with SDS, the concentration of the condensate has assumed a minimum.

The relationship between Triton X-114 boiling solution and condensate is shown in Fig.2(c). As can be seen from the figure, under the same concentration of the boiling solution, the concentration of Triton X-114 condensate rises with the increasing heat density, and a high heat flux increasing trend is particularly significant, like the Triton X-100. In the same heat flux density, Triton X-114 concentration condensate boiling solution with increasing concentration large, but also when the concentration CMC performance anomalies: contrast with SDS, the concentration of the condensate has assumed a minimum. 

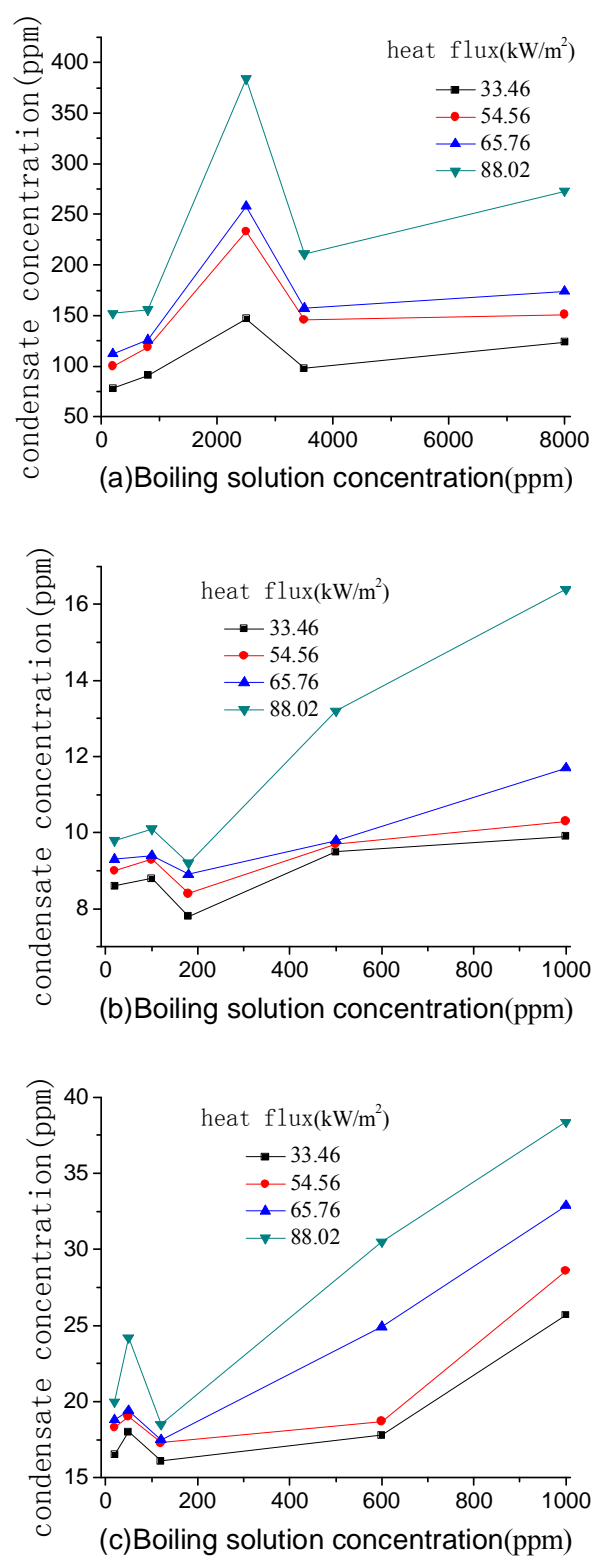

Figure3. The concentration relationship of SDS(a), TritonX-100(b), TritonX-114(c) boiling solution and condensate

\section{Analysis of Experimental Results}

From the experimental results, the mass distribution of aqueous surfactant solution is related to the factor of heat flux, surfactant solubility, boiling solution concentration and so on during the evaporation process.

The larger the heat flux, the more amount of surfactant solution is carried by the steam during evaporation. The main reason is that the larger the heat flux, the higher the steam humidity. The more water droplets carried by steam, the more surfactant dissolved in them is carried out. This growing trend at high heat flux is particularly significant. This is mainly because of a sharp increase of steam humidity at high heat flux. The reason is that under high heat flux, the number of bubbles and steam flow produce rapidly, which result to more water droplets and the larger of the bubble departure diameter, and more water droplets carried by the steam. So the steam humidity quickly increased.
Overall, the greater the concentration of boiling solution, the more amount of surfactant is carried out by the steam in the same heat flux. However, the condensate concentration exhibits an extreme at CMC, different types of surfactants showed the opposite extreme: Ionic surfactant SDS exhibits a maximum value, the non-ionic surfactant Triton X-100 and Triton X-114 exhibits a minimum value. This may due to the different relationships between solubility and temperature and different boiling solution concentration.

The solubility of SDS increases with the increasing of temperature. When reaches to Krafft point, solubility increases dramatically. At 2500ppm, the effect of nuclear pool boiling heat transfer enhancement by SDS reaches best. At this point, the boiling phenomenon is particularly violent, the amount of steam is large and humidity is high. And the temperature is higher than Krafft point. So SDS is easily dissolved in boiling liquid and carried out largely by steam. Thus, SDS at 2500ppm shows a maximum concentration of the condensate.

The solubility of Triton X-100 and Triton X-114 decreases with the increasing of temperature. When the temperature reaches the cloud point, their solubility will sharply decrease and the solution becomes cloudy because of precipitation. At $180 \mathrm{ppm}$ and $120 \mathrm{ppm}$, the effect of nuclear pool boiling heat transfer enhancement by Triton X-100 and Triton X-114 reaches best, and the amount of steam is large and humidity is high. But temperature of the solution is higher than cloud point, which leads to the decrease of solubility and precipitate. So the surfactant carried out by steam is little. Therefore Triton X-100 and Triton $\mathrm{X}-114$ in $180 \mathrm{ppm}$ and $120 \mathrm{ppm}$ show the minimum concentration of the condensate.

\section{CONCLUSIONS}

1. In the same concentration of boiling solution, the amount of surfactant carried out by steam increases with the increasing of heat flux during the evaporation process and at high heat flux, the increasing tendency is particularly significant.

2. Overall, the condensate concentration of SDS, Triton X-100 and Triton X-114 increase with the increasing of boiling solution. However, there will be abnormal situation at CMC: the condensate concentration of SDS showed a maximum value, while Triton X-100 and Triton X-114 was minimum. This is mainly caused by the different surfactant solubility and boiling solution concentration.

3. At the same concentration and heat flux, the amount of Triton X-114 carried out by steam is larger than Triton $\mathrm{X}-100$ during the evaporation process. This may be related to the length of Polyoxyethylene chain (the EO chain) of Triton X-100 and Triton X-114.

\section{ACKNOWLEDGMENT}

The research work was supported by National Natural Science Foundation of China under Grant No. 21205051.

\section{REFERENCES}

[1] Yuanchen Cui. "Development of surfactants.Henan chemical industry,"China,vol.12,pp.2-4,1994.

[2] Bo-zhou Chen. "The general situation and suggestions for development of surfactants,'Hubei chemical industry,vol.2.pp.2023,1988 . 
[3] Hong-mei Ma and Zhi-liang Zhu. "The application and research of surfactants in chemical cleaning,"Cleaning world,vol.21(4),pp.2227,2005 .

[4] Cheng L Mewes D and Luke A. "Boiling phenomena with surfactants and polymeric additive:a state of the art review,"International Journal of Heat and Mass Transfer,vol.50(13/14),pp.2744-2771,2007.

[5] Zong-hu Lin,Jun Wang and Guo-min Gui. "Heat transfer intensification,"Beijing : Chemical industry press,2007.

[6] R.I. Elghanam, M.M.EL. Fawal and R. Abdel Aziz. "Experimental study of nucleate boiling heat transfer enhancement by using surfactant," Ain Shams Engineering Journal, vol.2011(2),pp.195209,2011
[7] Ling Hou,Yongji Song and Tianqing Liu. "The Enhancement of Additive on Boiling Heat Transfer of Glycol Mixture," Chemical Industry and Engineering, vol.17(6),pp.349-353.2000.

[8] Zi-cheng Hu,Jia-qiang Gu and Xin-nan Song. "Pool boiling heat transfer with addition of surfactants," Journal of Jiangsu University,vol.32(4),pp.405-409,2011.

[9] Xin-nan Song,Jia-qiang Gu and Zi-cheng Hu. "An experiment on nucleate pool boiling heat transfer of aqueous surfactant solution," Journal of Jiangsu University,vol.31(2),pp.184-188,2010.

[10] Yan Wang,Jing Wang and Fang-zheng Lu, "Investigation on the experiment of determination of the critical micelle concentration of sodium dodecyl sulfate," Laboratory Science, vol.15(3),pp.7072,2012 . 